\title{
Simulation, Measurement, Communication \& Evaluation of Measurement and Simulation Dual-mode Terminal
}

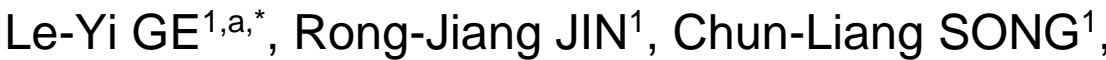 \\ Chao ZHANG ${ }^{1}$ and Zu-Qiang DENG ${ }^{1}$
}

\author{
'State Grid Corporation of China, \\ Electric Power Research Institute. \\ NARI Technology CO., LTD. \\ Beijing, 102200 \\ ageleyi@sgepri.sgcc.com.cn \\ ${ }^{*}$ Corresponding author
}

Keywords: Dual-mode terminal, Uncertainty, Real-time active power, Monte Carlo method.

\begin{abstract}
In this paper, measurement process and communication principle of measurement and simulation dual-mode terminal are introduced at first, then, a novel measurement uncertainty evaluation and parameter simulation method is described. According to analyzing the limited measurement results, we get probability characteristics of these data. And then, by big sample random simulations, sample space of these data can be expanded. Finally, in terms of the Bessel formula and the Uncertainty synthesis principle, measurement uncertainty can be evaluated rightfully.
\end{abstract}

\section{Introduction}

Because State Grid Corporation of China is making great efforts in developing smart distribution network construction, there are urgent needs of inventing a novel distribution network measurement and control terminal, which is a technology training tool of power enterprise distribution operation, fault simulation and simulation analysis.

Product developments for the distribution network measurement and control terminal (FTU, DTU, TTU) are relatively mature home and abroad. In the early 1980s, the terminal used 8051 single chip as the core chip, 485 serial as communication mode. Around the year 2000, the terminal used TMS320 DSP chips or ARM7 chip as the core, used the 485 serial, optical fiber cable network, GPRS as the main communication forms. In 2015, the terminal used ARM11, STM32 and MK60FN etc. as the core chips, used Wifi, 4G, mesh and optical fiber as communication forms. Product technology upgrade of measurement and control terminal focus on following aspects: measurement accuracy, communication form, the CPU frequency, flash size and so on. Latest technology of measurement and control terminal does not pay more attention to add signal simulation function to its core control board. namely according to commands of the remote master station technical workers or project maintenance personnel workers, the terminal can send the required data of telemetering, telecommunicating and telecontrolling in many communication forms. There are no such distribution network measurement and control terminal in the market home and abroad till the present moment.

Monte Carlo method is a numerical simulation method which is based on probability and statistics theory in use of random numbers [1]. Engineers and researchers home and abroad often use Monte Carlo method to solve many technical problems of power system. These studies, for example: Li Gengyin introduced a sequential Monte Carlo simulation approach for assessment of available transfer capability[2], Chen Houhe calculated probabilistic available transfer capability of wind farm incorporated system[3], Peng Hui used Monte Carlo Simulation method to comprehensive evaluate probabilistic security and probabilistic adequacy of composite power system[4] , furthermore, Monte Carlo method is used by Shi Luogang, Wang Xinyuan and Li 
Yudun to analyze the transmission capacity and load uncertainty of the wind farm power system[5-7].

For the measurement evaluation, GUM (Guide to expression of uncertainty in measurement) [8] is an universal standards home and abroad. The GUM analyzes all uncertainty source information of real-time measurement according to type B evaluation method, applies uncertainty propagation rule to the final measurement uncertainty calculation.

There are many researches about the distribution network measurement and control terminal, Monte Carlo method, measurement and control parameters simulation and application of GUM method, however, there is no new technology of measurement and simulation dual-mode terminal to combine these four aspects home and abroad.

In this paper, measurement process and communication principle of measurement and simulation dual-mode terminal were analyzed at first, we synthesized advantages of the Monte Carlo method and GUM method, and realized measurement uncertainty evaluation and parameters simulation. The measurement and simulation dual-mode terminal described in this paper can provide a new and effective way for distribution network information collection, electrical maintenance personnel training of State Grid Corporation of China.

\section{Measurement Process and Communication Mechanism}

\section{Measurement Process of Measurement and Simulation Dual-mode Terminal}

Measurement process of measurement and simulation dual-mode terminal can be shown in Figure 1. Signals which come from environmental parameter sensors, voltage transformers, current transformers and switches of distribution power grid are measured at first, the measured data is conducted by signal procession module and MK60FN1M core board, and finally, we get the measured values of key parameters of distribution power grid.

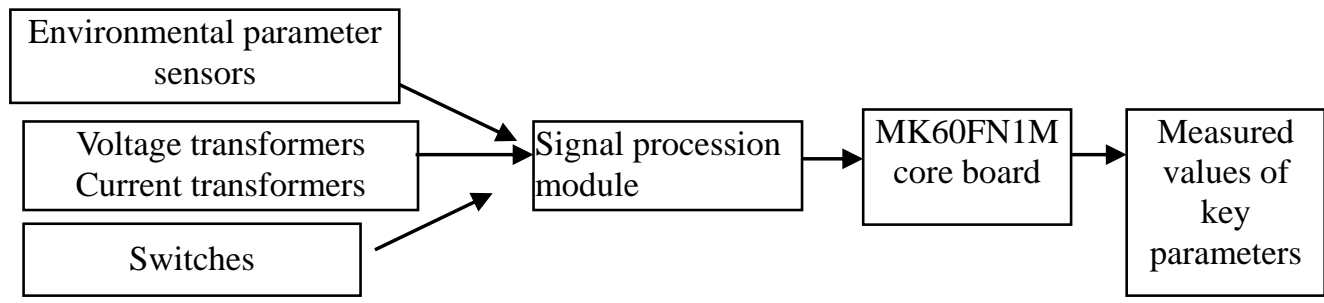

Figure 1. Measurement process of measurement and simulation dual-mode terminal

Data interfaces of measurement and simulation dual-mode terminal include following types: Digital input/ Digital output interface, Analog input/ Analog output interface, Inter-integrated circuit interface, Serial peripheral interface, Time to live interface, Universal serial bus interface, 232/485 serial interface.

\section{Communication Principle of Measurement and Simulation Dual-mode Terminal}

Uplink communication modes of measurement and simulation dual-mode terminal include Wifi, GPRS, 4G, Mesh (LTE230 wireless communication). Measurement and simulation dual-mode terminal is connected with public base network station (by GPRS, 4G) and private base network station (Wifi, Mesh). The public base network station and private base network station communicate with remote master station of distribution automation system in use of Ethernet. The terminal can send the required data of telemetering, telecommunicating and telecontrolling to the remote master station. In addition, the terminal can be connected with remote master station by optical fiber.

Downlink communication of measurement and simulation of dual-mode terminal use following data interfaces: Digital input/ Digital output interface, Analog input/ Analog output interface, Inter-integrated circuit interface, Serial peripheral interface, Time to live interface, Universal serial bus interface, 232/485 serial interface. The measurement and simulation of dual-mode terminal can 
exchange data with various switches of distribution power grid, environmental sensors and electrical sensors. Uplink and downlink communication of the terminal can be shown in Figure 2 below:

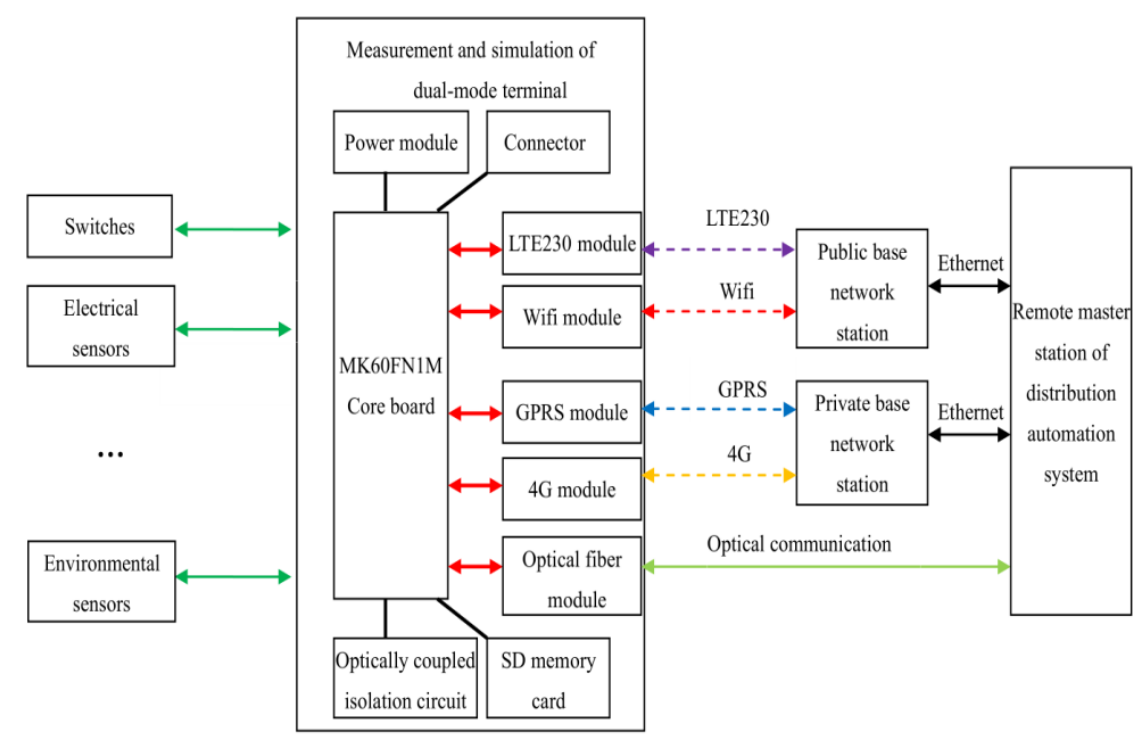

Figure 2. Uplink and downlink communication of measurement and simulation of dual-mode terminal

\section{Simulation Mechanism and Uncertainty Evaluation}

\section{Simulation Mechanism of Measurement and Simulation Dual-Mode Terminal}

The simulation process of measurement and simulation dual-mode terminal is carried out according to three steps, as shown in Figure 3:

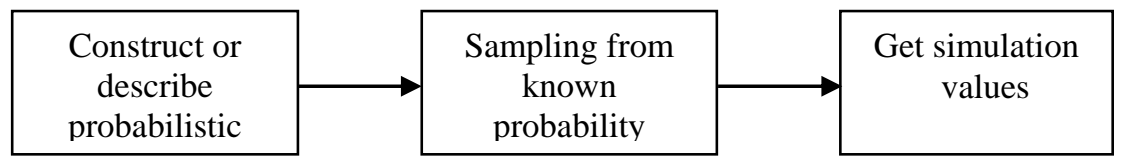

Figure 3. Simulation mechanism of measurement and simulation dual-mode terminal

At first, we establish a random test model for distribution grid device running states, measurement states and control states, different random test models need to be established according to different technical problems. Sampling from known probability refers to run randomized trials, use random variables of the measured parameters in the random model as inputs, in terms of Monte Carlo simulation method, generate corresponding simulation output. The simulation results show this method's calculation amount is small, convergence of the speed is fast, and the simulation values are estimated accurately.

\section{Measurement Uncertainty Evaluation Principle of Measurement and Simulation Dual-Mode Terminal}

According to Monte Carlo method, pseudo random in the interval [0,1] which obeys uniform distribution and passes randomness tests is generated. The more randomness tests the pseudo-random numbers pass, the better the randomness of the pseudo-random numbers. Probability distribution information (distribution range and distribution type) is established in terms of measurement results of the measurement and simulation dual-mode terminal. Lots of random are generated by random sampling in this probability distribution range to simulate corresponding measurement results. We execute Monte Carlo simulation for $\mathrm{n}$ times, obtain $\mathrm{n}$ simulation data of measurement results of the measurement and simulation dual-mode terminal, carry out statistical 
analysis and get standard deviation, the standard deviation represents standard measurement uncertainty of the measurement and simulation dual-mode terminal. Measurement uncertainty evaluation principle of measurement and simulation dual-mode terminal is described in following Figure 4.

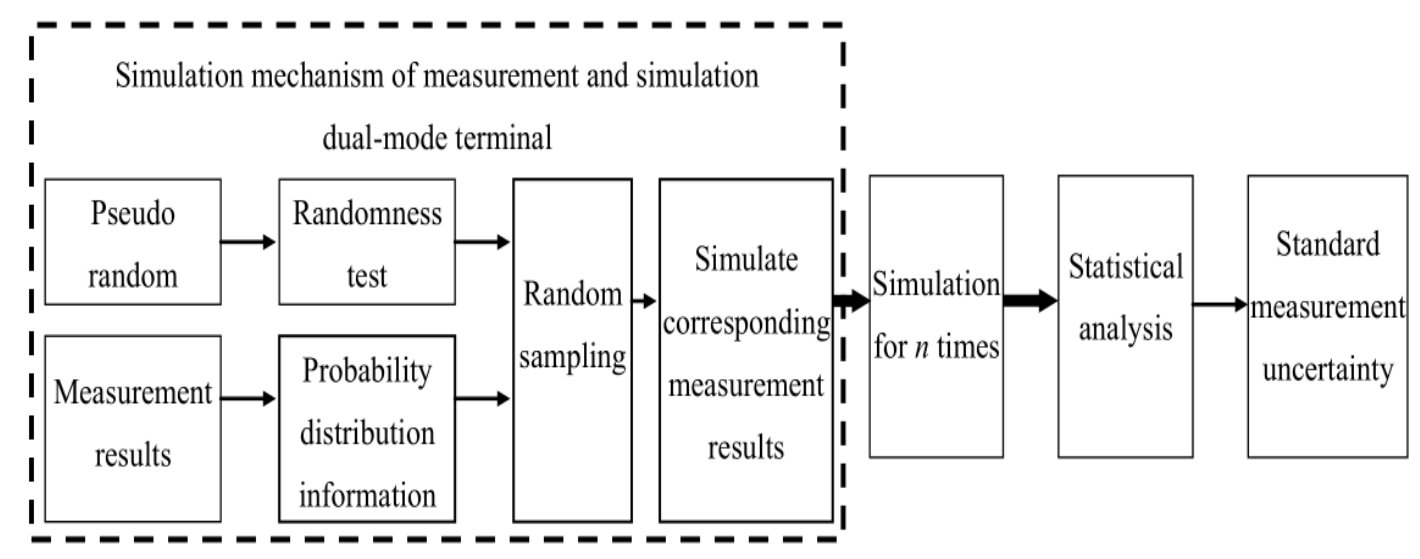

Figure 4. Measurement uncertainty evaluation principle of measurement and simulation dual-mode terminal

Several key problems involved in simulation mechanism and uncertainty evaluation based on Monte Carlo method of measurement and simulation dual-mode terminal are following aspects:

1) How to analyze probability distribution of measurement results on the basis of the measurement results sample space and sensor characteristics;

2) How to pseudo random sample according to the specific probability characteristics, to expand the sample space of measurement results.

\section{Summary}

In this paper, a novel measurement and simulation of dual-mode terminal is proposed, its measurement process, communication mechanism, simulation principle and evaluation method are analyzed in detail. innovation points of the measurement and simulation of dual-mode terminal can be summed up as following aspects:

(1) In use of a low-cost 32-bit ARM cortex M4 as the processor, measurement and simulation of dual-mode terminal integrates several communication modes to realize data communication and interaction for the switches, environmental sensors, electrical sensors and Remote master station of distribution automation system.

(2) Based on small size sample of measurement error calibration data, we analyze probability distribution of the data, and generate corresponding large sample size of pseudo random number to simulate measurement process. The method can broaden the data sample space, and then realize measurement uncertainty evaluation for the measurement and simulation of dual-mode terminal in terms of guide to expression of uncertainty in measurement.

\section{Reference}

1. WANG Zhongyu. Novel uncertainty-evaluation method of virtual instrument small sample size[J]. Journal of Testing and Evaluation. 2008,36(3): 101454-101461.

2. LI Gengyin, GAO Yajing, ZHOU Ming. Sequential Monte Carlo Simulation Approach for Assessment of Available Transfer Capability [J]. Proceedings of the CSEE.2008,28(25): 74-78. 
3. CHEN Houhe, LI Guoqing, ZHANG Fangjing. Calculation of probabilistic available transfer capability of wind farm incorporated system [J]. Power System Protection and Control.2014,42(21):59-64.

4. PENG Hui, LIANG Wenju, ZHANG Xin. Probabilistic security and probabilistic adequacy comprehensive evaluation of composite power system[J].Advanced Technology of Electrical Engineering and Energy.2014,33(9):41-46.

5. LUO Gang,SHI Dongyuan,CAI Defu. Fast calculation of probabilistic available transfer capability considering correlation in wind power integrated systems[J].Proceedings of the CSEE.2014,34(7):1024-1032.

6. WANG Xinyuan, GUO Ruipeng. Reliability assessment of power system with large-scale wind power integration considering load uncertainty[J].East China Electric Power.2013,41(2):385-389.

7. LI Yudun. Reliability assessment of power systems containing multiple wind farms [J].Journal of Electric Power Science And Technology.2011, 26(1): 73-76.

8. Guide to expression of uncertainty in measurement[S]. ISO, 1995.

9. DU Haixia.Evaluation of measurement uncertainty for ratio difference of the current mutual inductor[J].Inner Mongulia Petrochemical Industry. 2013(21):94-95. 